\title{
Erratum to: A Preliminary Study of an Integrated and Culturally Attuned Cognitive Behavioral Group Treatment for Chinese Problem Gamblers in Hong Kong
}

\author{
Daniel Fu Keung Wong • Catherine Lai Ping Chung • Janet Wu • \\ Joe Yiu Cho Tang • Patrick Tak Ching Lau · Jennie Po Ching Wan
}

Published online: 1 June 2014

(C) Springer Science+Business Media New York 2014

\section{Erratum to: J Gambl Stud DOI 10.1007/s10899-014-9457-2}

Unfortunately, Jennie Po Ching Wan was not included as one of the authors in the original publication of this article. The correct and complete order of authors is provided in this erratum. Her affiliation is provided below.

The online version of the original article can be found under doi:10.1007/s10899-014-9457-2.

D. F. K. Wong $(\bowtie) \cdot$ J. Wu

Department of Applied Social Studies, City University of Hong Kong, Tat Chee Avenue,

Kowloon Tong, Kowloon, Hong Kong

e-mail: dfk.wong@cityu.edu.hk

J. Wu

e-mail: shuiswu@cityu.edu.hk

C. L. P. Chung

Department of Social Work and Social Administration, University of Hong Kong, Pokfulam Road, Pokfulam, Hong Kong

e-mail: catchung@hku.hk

J. Y. C. Tang $\cdot$ P. T. C. Lau $\cdot$ J. P. C. Wan

Caritas Addicted Gamblers Counselling Centre, Caritas-Hong Kong, Room 1708-9 Chinachem Tsuen Wan Plaza, 455-457 Castle Peak Road, Tsuen Wan, N.T., Hong Kong

e-mail: tangjoe@caritassws.org.hk

P. T. C. Lau

e-mail: laupatrick@caritassws.org.hk

J. P. C. Wan

e-mail: wanjennie@ caritassws.org.hk 